1 National Institute for Health and Care Excellence, London, UK

2 Guildhall and Barrow Surgery, Bury St Edmunds IP33 1ET, and Primary Care Unit, Department of Public Health and Primary Care, Cambridge University, UK

Correspondence to: C Carmona sdmguideline@nice.nhs.uk Cite this as: BMJ 2021;373:n1430 http://dx.doi.org/10.1136/bmj.n1430 Published: 17 June 2021

\title{
GUIDELINES
}

\section{Shared decision-making: summary of NICE guidance}

\section{Chris Carmona, ${ }^{1}$ Joseph Crutwell, ${ }^{1}$ Marie Burnham, ${ }^{1}$ Louisa Polak ${ }^{2}$, on behalf of the Guideline Committee}

\section{What you need to know}

- Shared decision-making requires organisational leadership and planning as well as practitioner skills

- Shared decision-making is a process requiring a collaborative relationship between patient and healthcare professional; it is not a one-off intervention that healthcare professionals can insert into the consultation

- Use patient decision aids as part of a toolkit to support shared decision-making

- Discuss risks, benefits, and consequences of different options in the context of the person's life and values

- Be aware that different people interpret terms such as "risk," "rare," "unusual," and "common" in different ways

Shared decision-making is widely accepted as a core feature of good healthcare. Principle 4 of the NHS constitution states that "Patients, with their families and carers, where appropriate, will be involved in and consulted on all decisions about their care and treatment." ${ }^{1}$ Because most NHS practitioners and commissioners acknowledge that this involvement and consultation is not always offered, the National Institute for Health and Care Excellence (NICE) was asked to produce guidance about facilitating shared decision-making and embedding it in everyday practice. For the purposes of the guideline, shared decision-making was defined as "a collaborative process that involves a person and their healthcare professional working together to reach a joint decision about care."

This article summarises the recommendations from the NICE guideline. ${ }^{2}$

\section{Recommendations}

NICE recommendations are based on systematic reviews of best available evidence. When minimal evidence is available, recommendations are based on the Guideline Committee's experience and opinion of what constitutes good practice. Evidence levels for the recommendations are given in italic in square brackets.

\section{Embedding shared decision-making at an organisational level}

Shared decision-making is more likely to become standard practice in organisations when it is led from the highest levels of the organisation. This can drive systematic planning and implementation of shared decision-making across the organisation. A dual approach is needed, promoting shared decision-making to people who use services as well as training and supporting staff to deliver it.
The first set of guideline recommendations aims to support this approach in all sizes of healthcare organisation, with recommendations about high level leadership; planning and implementing shared decision-making; sharing information between services; encouraging healthcare professionals to develop and maintain skills and competencies; and promoting shared decision-making to people who use services.

\section{Putting shared decision-making into practice}

The guideline recommends things that healthcare professionals can do before, during, and after discussions with patients and service users to facilitate shared decision-making. This includes making sure that information sources are reliable and of high quality, and that they are likely to be accessible to the people for whom they are intended.

\section{Before an appointment}

When possible, shared decision-making should begin in advance of any discussion or appointment to maximise the person's ability to participate and to reassure them that shared decision-making will be supported by the healthcare professional they see.

- Offer the person access to resources that encourage them to think about what matters to them, what they hope will happen as a result of the discussion, and what questions they would like to ask. [Based on moderate to very low quality randomised controlled trial evidence and the experience and opinion of the Guideline Committee $(G C)]$

- Ask the person if they would like to invite a friend or relative to join the discussion, in order to help them understand the resources provided and support them to take an active part in decision-making. [Based on moderate to very low randomised controlled trial evidence and the experience and opinion of the GC]

- For people who do not have anyone they would like to ask to support them, and who might find it difficult to share in decision-making, offer additional support-for example, from a nurse, social worker, translator, or volunteer. [Based on moderate to very low randomised controlled trial evidence and the experience and opinion of the GC]

\section{During an appointment}

- Create a collaborative atmosphere: agree an agenda for the conversation; make sure patients or service users understand that they can participate as much as they want; encourage people to think about what matters to them; allow 
enough time to answer questions; and offer a further opportunity for discussion. [Based on moderate to very low randomised controlled trial evidence and the experience and opinion of the GC]

- Discuss the risks, benefits, and consequences of the possible tests, treatments, or interventions openly: clarify what the person hopes to gain from the intervention and discuss their ideas and concerns. Explain the potential benefits and harms of each option, including doing nothing. [Based on moderate to very low randomised controlled trial evidence and the experience and opinion of the $G C$ ]

- Make a record of the discussion (for example, in the clinical notes or care plan) that includes any decisions made along with details of what the person said was important to them in making those decisions. Share this with the person, for example, in a post-clinic letter (letters should be written directly to patients and copied to the relevant healthcare professionals). [Based on the experience and opinion of the GC]

\section{Following an appointment}

The guideline committee also recommended actions to follow up from appointments.

- Offer people resources to help them understand what was discussed and agreed. This could be a printout summarising their diagnosis, the options and decisions or plans made, and links to high quality online resources options. [Based on moderate to very low randomised controlled trial evidence and the experience and opinion of the $\mathrm{GC}$ ]

- Ensure that information provided after discussions includes details of who to contact with any further questions. [Based on the experience and opinion of the $G C]$

- Offer additional support to people who are likely to need extra help to engage in shared decision-making. This could include encouraging them to record the discussion, explaining in writing the decisions that have been made, or arranging follow-up by a clinical member of staff or a suitable alternative. [Based on the experience and opinion of the GC]

\section{Patient decision aids}

The recommendations focus on the need for patient decision aids (PDAs) to be quality assured. They also stress that PDAs are a tool to support shared decision-making and that shared decision-making is not dependent on them.

The committee recommended the following actions for PDAs in shared decision-making.

- Use patient decision aids as one part of an overall "toolkit" to support shared decision-making, alongside the other skills and interventions outlined in the guideline.

- Only use a patient decision aid if it is:

- Up to date and reflects evidence-based best practice

- Relevant to that discussion, and the decision that needs to be made

- Relevant to that clinical setting.

- Before using a particular decision aid, healthcare professionals should be familiar with it, including how it will help people to understand which option is best for them.
The committee also made recommendations for organisations to maintain a database of decision aids that are regularly reviewed and updated, or signpost staff to decision aids produced by national bodies such as NICE, and to ensure they are available in different ways to support people's needs (for example, in print, online, or in different languages). [PDA recommendations based on previous NICE recommendations, evidence from randomised controlled trials, and the experience and opinion of the $G C]$

\section{Risk communication}

Finally, the committee updated the section on risk communication from the NICE guideline on patient experience in adult NHS services ${ }^{3}$ and brought it into the shared decision-making guideline. Rather than referring to risk communication, the committee agreed it was better to talk to patients about "risks, benefits, and consequences" since people often interpret the term "risk" as negative. They recommended that healthcare professionals:

- Discuss risks, benefits, and consequences in the context of each person's life and what matters to them.

- Personalise information on risks, benefits, and consequences as much as possible.

- Make it clear to people how the information they are providing applies to that person personally and how much uncertainty is associated with it.

- Have a good understanding of the information and how to apply and explain it clearly.

The committee also made a series of recommendations about the best ways to communicate numerical information to people They agreed that this would vary according to the person but that generally it was best to:

- Use a mixture of numbers and pictures (for example, numerical rates and pictograms or icon arrays) to allow people to see both positive and negative framing at the same time.

- Use numerical data to describe risks if available (different people interpret terms such as “risk," "rare," "unusual," and “common" in different ways).

- Use absolute risk rather than relative risk and natural frequencies (for example, 10 in 100) rather than percentages (10\%).

- Be consistent when using data. For example, use the same denominator when comparing risk.

- Present a risk over a defined period of time (months or years) if relevant. For example, if 100 people are treated for 1 year, 10 will experience a given side effect.

- Use both positive and negative framing. For example, treatment will be successful for 97 out of 100 people and it will be unsuccessful for 3 out of 100 people.

[Risk communication recommendations based on previous NICE recommendations, evidence from systematic reviews, and the experience and opinion of the $G C]$

\section{Implementation}

The Guideline Committee was aware that implementing shared decision-making is a complex task that needs to be led from the highest levels of the organisation, supporting practitioners to facilitate shared decision-making in every clinical encounter. 
The committee agreed that recommendations might need to be tailored to specific circumstances-for example, it is not always possible to provide people with information or resources before a discussion if the appointment is urgent or the healthcare professional does not know what the content of the discussion will be. The committee also noted that, in some healthcare settings, appointments or consultations may need to be longer, and this could represent a substantial resource impact, but might lead to fewer subsequent appointments and will ensure that the right decisions are made with people. The committee also noted that there is currently no national-level repository of patient decision aids, which makes it harder for healthcare professionals to identify and use high quality evidence-based decision aids.

\section{Future research}

The Guideline Committee made a series of recommendations for research.

- How do the same shared decision-making interventions differ in effectiveness between different groups of people and different care settings

- How best to measure the effectiveness of shared decision-making in different contexts

- How organisations can sustain shared decision-making once they have embedded it.

- How to encourage people to engage in shared decision-making if they see healthcare professionals as "authority figures"

- How shared decision-making might need to be modified for remote consultations (for example, by telephone or online)

\section{Guidelines into practice}

- As a healthcare professional in a busy setting with limited continuity of care, how can you promote shared decision-making within your everyday practice?

- When you organise or commission clinical services, how can you ensure that practitioners' working arrangements support them to practise shared decision-making, and that they are competent to do so?

\section{How patients were involved in the creation of this article}

Committee members involved in this guideline update included lay members who contributed to the formulation of the recommendations summarised here.

\section{Further information on the guidance}

This guidance was developed by the Guideline Updates Team within NICE in accordance with NICE guideline methodology (www.nice.org.uk/me$\mathrm{dia} /$ default/about/what-we-do/our-programmes/developing-niceguidelines-the-manual.pdf). A guideline committee (GC) was established which represented a broad range healthcare and allied healthcare professionals (including dentistry), academics, and lay members.

The guideline is available at https://www.nice.org.uk/guidance/ng197 The GC identified relevant review questions and collected and appraised clinical evidence. Quality ratings of the evidence were based on GRADE methodology (www.gradeworkinggroup.org). These relate to the quality of the available evidence for assessed outcomes or themes rather than the quality of the study. The GC agreed recommendations for practice based on the available evidence or, when evidence was not found, based on their experience and opinion using informal consensus methods. The scope and the draft of the guideline went through a rigorous reviewing process in which stakeholder organisations were invited to comment. The GC took all comments into consideration when producing the final version of the guideline.
NICE will conduct regular reviews after publication of the guidance, to determine whether the evidence base has progressed sufficiently to alter the current guideline recommendations and require an update.

Contributors: CC drafted the summary and is responsible for the overall content as guarantor. JC undertook the systematic reviewing and authorship of the technical documents underpinning the full guideline and was a collaborator in drafting this summary. MB was chair, and LP a member, of the guideline committee that interpreted the evidence and made the recommendations contained in the guideline. They have been collaborators in producing this summary on behalf of the guideline committee.

Competing interests: We declare the following interests based on NICE's policy on conflicts of interests (https://www.nice.org.uk/Media/Default/About/Who-we-are/Policies-and-procedures/declaration-ofinterests-policy.pdf). CC and JC are employees of the National Institute for Health and Care Excellence. MB and LP provide full statements in the NICE guideline, which can be viewed along with all the guideline authors at https://www.nice.org.uk/guidance/ng197.

Funding: CC and JC are employees of NICE, which is funded by the Department of Health and Social Care to produce guidelines. MB and LP were not funded to collaborate in producing this summary.

The members of the Guideline Committee were (alphabetically): Nina Barnett, Marie Burnham (chair), Adrian Edwards, Gilli Erez, Paul Hutton, David Metcalfe, Helen Morgan, Sian Phipps, Carol Pitkeathley, Louisa Polak, Joshua Ruegger, David Shaw, Chloe Stewart, Wendy Thompson, Richard Thomson, Nige Westwood, Georgina Wiley

Department of Health and Social Science. NHS constitution for England. 2012, updated 2021. https://www.gov.uk/government/publications/the-nhs-constitution-for-england.

2 National Institute for Health and Care Excellence. Shared Decision Making (NICE guideline ng197). 2021. www.nice.org.uk/guidance/ng197.

3 National Institute for Health and Care Excellence. Patient experience in adult NHS services: improving the experience of care for people using adult NHS services (Clinical guideline CG138). 2012. https://www.nice.org.uk/guidance/cg138. 